\title{
Fibromyalgia—all in the brain?
}

$\mathrm{F}$ ibromyalgia, a condition characterized by chronic widespread pain, tenderness and fatigue, markedly impairs the quality of life of affected patients. Knowledge of its pathogenesis might help us to prevent or treat the disease, but our understanding of the underlying mechanisms is currently limited. Prior neuroimaging studies have revealed alterations in brain function in patients with fibromyalgia, as well as accelerated grey-matter loss. "However, the relationship between altered brain function and structure in fibromyalgia was unknown," says Karin Jensen, introducing research that now provides insight into this connection.

The new study, in 26 female patients with fibromyalgia (24-48 years of age) and 13 age-matched healthy female controls, used MRI and functional MRI (fMRI) to explore brain abnormalities associated with fibromyalgia. Treatments that can influence pain perception were not permitted during the study, although NSAIDs were allowed as rescue medication. Correlations of brain abnormalities with depression scores and fibromyalgia duration were also investigated, to provide clues to the underlying mechanisms.

In accordance with previous work, patients with fibromyalgia had significantly lower total (supratentorial) brain volumes than controls $(P=0.001)$. Examining this finding in more detail, the investigators showed that the cortical thickness and volume of several brain regions, including the left rostral anterior cingulate cortex ( $\mathrm{rACC})$, were reduced in the patients compared with the controls.

"The ACC is an important centre in the medial pain pathway and has a pivotal role in pain processing by integrating the sensory, attentional and emotional components of pain," explains Professor Robert Bennett, a fibromyalgia expert from Oregon Health and Science University, USA, who was not involved in the study. The structural alterations in the rACC and in total brain volume correlated with fibromyalgia duration, suggesting that such changes might occur over time in response to pain, although the authors emphasize that further longitudinal studies will be required to confirm this hypothesis. Thus, whether these abnormalities are present at disease onset and have a causative role in the pathogenesis remains unclear.

In the fMRI part of the study, the researchers used the regional homogeneity method, which measures the local synchrony of brain activity, to assess functional connectivity over a 16-minute time period, during which intermittent, painful pressure stimuli were applied. The stimuli were adjusted for each participant in accordance with their pain sensitivity, as determined by visual analogue scale scores obtained during a calibration experiment. Interestingly, in view of the anatomical results for the rACC, patients with fibromyalgia displayed significantly decreased regional coherence in this brain area compared with controls $(P=0.05)$. Moreover, a correlation was observed between rACC volume and coherence measures for each patient, indicating that atrophy of this region might be associated with diminished functional connectivity.

Given the known role of the rACC in descending pain modulation, the results endorse the idea that dysfunction in this system is linked to the pathogenesis of fibromyalgia. "The connection between decreased cortical thickness and measures of coherence in the ACC is an original and important finding," enthuses Bennett. Nonetheless, he points out that the fMRI signals occurred in response to pain stimuli; "thus they do not provide any new insights into the 'resting' brain of patients with fibromyalgia," he cautions.

In further analyses, associations were revealed between depression and several anatomical and functional brain alterations. However, the changes in the rACC did not correlate with depression, suggesting that pain and depression are associated with independent neuroanatomical changes.

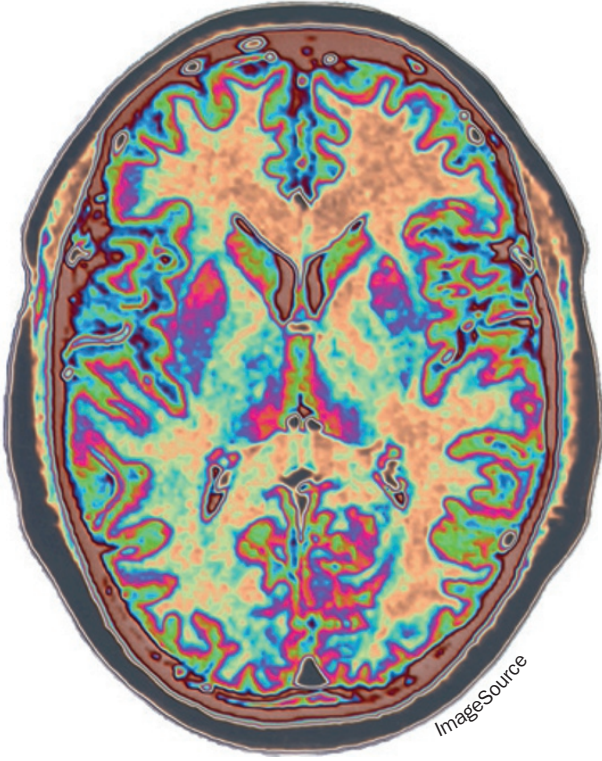

"The next step is for these findings to be independently confirmed", asserts Bennett. "As always a larger sample will be necessary," adds Professor Roland Staud, from the University of Florida, USA, who also advocates correcting for the multiple statistical comparisons to confirm the reported differences. "With more statistical power, additional brain areas besides the rACC may become detectable that are involved in pain facilitation and inhibition," he continues. "Furthermore, ... alternative explanations for the rACC abnormalities of patients with fibromyalgia need to be explored."

In the longer term, Jensen hopes the findings will provide a platform for further research advances. "With specific hypotheses about brain regions that reflect pain pathology, we would like to use the combination of structural and functional brain measures to find early indicators of pain chronification," she proposes. "Ultimately, we hope that the identification of brain regions affected by fibromyalgia will lead to early prediction and prevention of chronic pain."

Isabel Woodman

Original article Jensen, K. B. et al. Overlapping structural and functional brain changes in patients with long-term exposure to fibromyalgia. Arthritis Rheum. doi:10.1002/ art.38170 